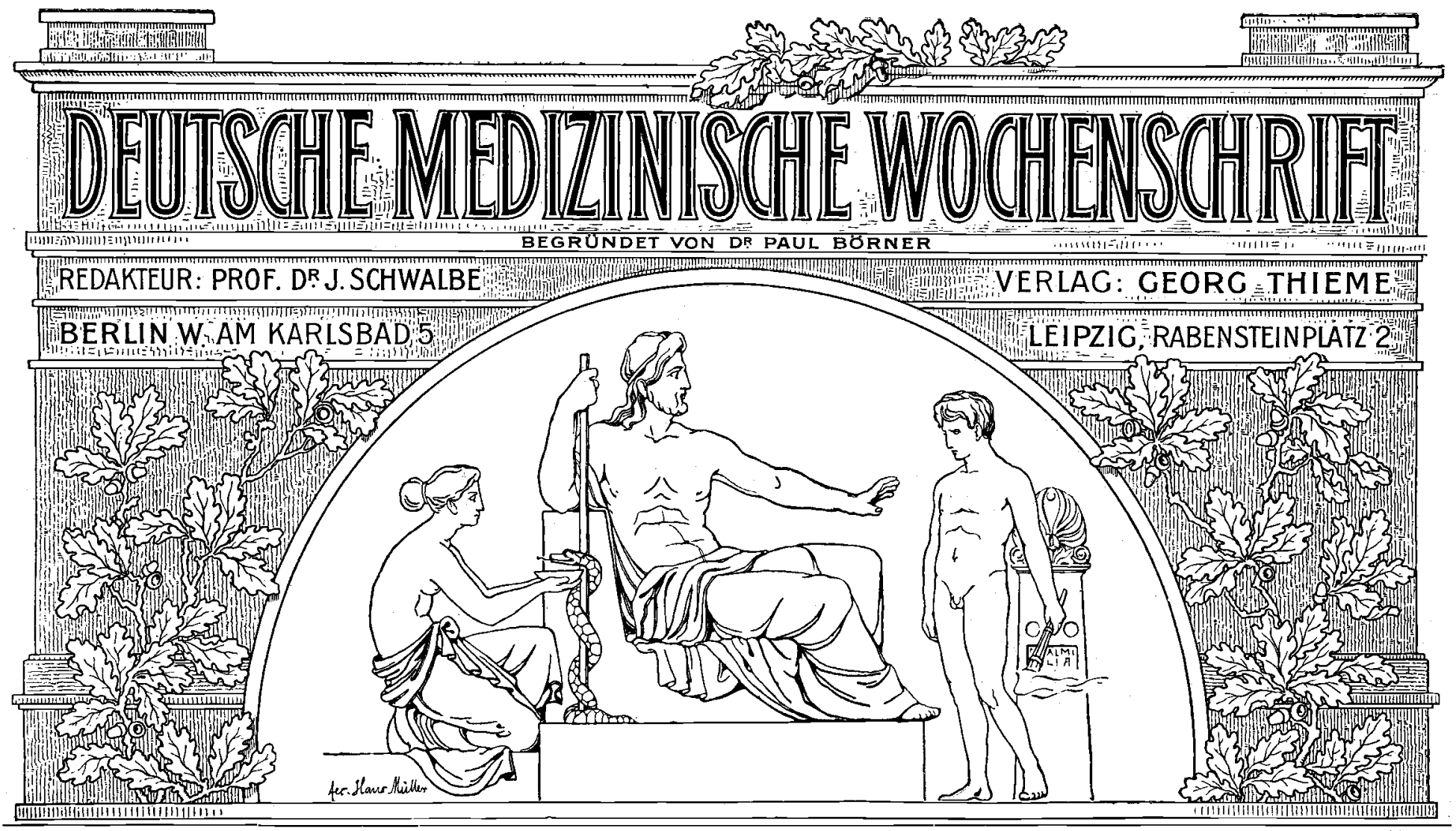

No. 8.

Donnerstag, den 21. Februar 1907.

33. Jahrgang.

\section{Die natürliche Säuglingsernährung in der ärztlichen Praxis.}

Fortbildungsvortrag.

Von Priv.-Doz. Dr. H. Neumann in Berlin.

M. H.! Sie sind über den Vorzug der natürlichen Ernährung zu gut unterrichtet, als daß Sie von mir viel mehr als Binsenwahrheiten erwarten werden. Trotzdem bitte ich Sie, mir für einige Minuten Ihr Ohr zu schenken, zumal eine Aussprache nicht nur mit dem Praktiker, sondern auch mit dem Geburtshelfer an dieser hervorragenden Stelle möglich ist.

Mit der überwiegenden Mehrzahl der Geburten hat leider weder der eine noch der andere von Ihnen zu tun, da sich der unbemittelte Teil der Bevölkerung, außer in pathologischen Fällen, nur der Hilfe der Hebammen bedient. Es steht dies ja z. B. in Paris anders, wo ein großer Teil aller Geburten in den Gebäranstalten stattfindet. Ich denke daher bei diesen Ausführungen wesentlich an die Geburten beim Mittelstand und bei den Wohlhabenden. Nehme ich die Größe der Wohnungen als Maßstab, so handelt es sich um Wohnungen von vier und mehr Räumen Bekanntlich nähren in Berlin - wie es wohl auch in anderen Städten sein wird - die Bessergestellten ihre Kinder noch seltener als die Unbemittelten. Aber es wurde bisher noch nicht betont, daß sich auch der Rückgang im Stillen, den wir in Berlin seit 1885 bei jeder Volkszählung feststellen, auch auf den kleineren Kreis der Bevölkerung, von dem wir hier sprechen, bezieht. Der Prozentsatz der künstlich ernährten Säuglinge hat sich bei den Bessergestellten wie bei der Gesamtbevölkerung von dem Jahre 1885 bis zum Jahre 1900 um fast $2 / 3$ vermehrt. Dieser Tatsache gegenüber ist die Frage berechtigt, ob wir in der künstlichen Ernährung wirklich so weit fortgeschritten sind, um ihre zunehmende Ausbreitung unbesorgt vor sich gehen zu lassen.

DaB sich das Prinzip der Portionsflaschen, das Soxhlet eingeführt hat, ungemein wertvoll erwiesen hat, ist nicht zu bezweifeln. Nachdem es die äußeren Formen, an die es zunächst geknüpft war, abgestreift hat und nachdem die übertriebene Erhitzung aufgegeben ist, können wir dies gern anerkennen. Mindestens ebenso wichtig ist aber die gute $\mathrm{Be}$ schaffenheit der Milch, wofür der Teil der Bevölkerung, von dem ich hier spreche, durch Aufwendung eines höheren Preises (30-60 Pf.) leicht sorgen kann. Da auch das Wohnungselend in seinem ungünstigen Einfluß auf die Sommerdurchfälle hier außer Betracht bleibt, so kommt bei den Bessergestellten eine akute Darmstörung nur noch recht selten vor und führt nur ganz ausnahmsweise zum Tode. ${ }^{\text {) }}$

Schon viel weniger sicher lassen sich selbst unter günstigen äußeren Verhältnissen bei künstlicher Ernährung lang. sam verlaufende Darmstörungen vermeiden. Es sind die Fortschritte der allerletzten Jahre in der Säuglingsernährung auch noch viel zu wenig bekannt, um schon einen Einflub auf die Diätetik ausüben zu können. Man wagt es noch nicht, in Größe, Häufigkeit und Zusammensetzung der Mahlzeit die durch die Ernährungsstörung gebotenen Anzeigen einzuhalten; man glaubt noch: je mehr Nahrung, um so besser, und man probiert aufs Geratewohl, in der Erwartung, schlieflich durch einen Zufall auf das für den einzelnen Fall geeignete Nährmittel zu stoßen. Der eine Arzt schwört auf ein bestimmtes, weitgepriesenes Nährmittel, der andere mischt als Eklektiker eine gröbere Zahl zusammen. ${ }^{2}$ ) Trotz alledem entwickeln sich aus leichten Störungen - Speien, Brechen, Verstopfung allmählich hartnäckige Ernährungsstörungen, welche auf Jahre hinaus die Entwicklung schädigen können.

Solche Mißerfolge sehen wir schon bei der künstlichen Ernährung normal entwickelter Säuglinge, und zwar werden sie um so bedenklicher, je früher das Kind mit seiner Verdauung in Unordnung kommt. Selbst die nachträgliche Einleitung natürlicher Ernährung bringt die allmählich herangezüchtete Störung nicht immer ohne weiteres zum Schwinden, sondern es kann auch hierbei noch wochenlang die vorsichtigste Diätetik nötig sein und während des Reparationsstadiums eine Gewichtszunahme auf sich warten lassen. ${ }^{3}$ ) Immerhin führt die künstliche Aufzucht normal entwickelter Neugeborener in vielen Fällen zu einem befriedigenden Ergebnis, wenn die Verdauungsorgane von Natur kräftig sind. Unter Umständen können wir die familiäre Anlage nach

1) Im Jahr 1904 kamen von 3186 'Todesfällen' an Darmkrank heiten bei Säuglingen in Wohnungen von vier Zimmern und Küche nur 23, in größeren Wohnungen nur 13 Fälle vor.

$\left.{ }^{2}\right)$ Ich fand kürzlich bei einem Kind von zehn Monaten folgende Nahrung. Fin Liter Victoriaparkmilch, fünf Teelöffel Ramogen, 1/2 Teelöffel Roborat, 1/2 Teelöffel Eisenzucker, zwei Eßlöffel Mellins Food und zwei EBlöffel Milchzucker. Das Kind litt an ,Milchnährschaden" (nach Keller und $\mathrm{Czerny}$ ) und nalm die Nahrung nur schwer zu sich; statt ihrer wurde viermal Malzsuppe und eine gemischte Mahlzeit verordnet.

3) Vgl. Czerny und Keller, Des Kindes Ernährung, Ernährungsstörungen und Ernăhrungstherapie 1906, 1. Bd. 1.eipzig-Wien, Franz Deuticke. 
den Ërnährungserfolgen bei früheren Kindern und hiernach die Dringlichkeit der natürlichen Ernährung im vorliegenden Fall abschätzen. Bei unterwertigen Neugeborenen - Frühgeburten oder sehr leichten Zwillingen - ist die Flaschenernährung hingegen unbedingt $\mathrm{zu}$ verwerfen. Es ist garnicht zu leugnen, daß man auch hier mit den verschiedensten Methoden (z. B. mit Buttermilch) die schönsten Gewichtszunahmen erzielen kann; aber hiermit ist keineswegs eine gesunde Entwicklung gewährleistet.

Der Irrtum, das Gedeihen eines Säuglings und seinen augenblicklichen Gesundheitszustand an der Wage ablesen zu können, ist allgemein verbreitet und höchst gefährlicher Art, sodal ich ihn ausführlicher besprechen muß. Während der regelmäBigen Gewichtszunahmen können sich die schwersten Ernährungsstörungen vorbereiten. Bei jungen Säuglingen, vor allem bei unterwertigen, aber auch bei normal entwickelten, treten z. B. schwere Krämpfe auf, die nur bei Ersatz der Kuhmilch durch Ammenmilch wieder schwinden. Um ein Beispiel zu geben, so hatte eine Frühgeburt von $1600 \mathrm{~g}$ bei Backhausmilch in den ersten zehn Lebenswochen gut zugenommen (bis auf $2850 \mathrm{~g}$ ), dann traten dyspeptische Krämpfe auf, die nur durch Frauenmilch zum Verschwinden gebracht werden konnten. Solche Fälle sind nicht selten; in einem Fall bekam immer derjenige Zwilling, der aus Mangel an Ammenmilch künstlich ernährt wurde, Krämpfe, während der andere von ihnen verschont blieb, so lange er an der Brust war.

Ueberhaupt sind, wie ich hier einschalten will, bei Säuglingen jeden Alters alle starken Gewichtszunahmen über $250 \mathrm{~g}$ in der Woche - zu beanstanden, wenn sie sich während einer Reihe von Wochen wiederholen -- mag es sich um künstliche oder natürliche Ernährung handeln. Es gibt nur wenige Kinder, die gewöhnlich denn auch ein starkes Längenwachstum und sonst eine kräftige Entwicklung zeigen, deren Verdauungskanal die übermäßige Inanspruchnahme für die Dauer aushält. Gewöhnlich folgt auf die schöne Zeit der großen Zunahmen die Zeit des Schwankens, Stillstandes oder Rückganges. Auch dann kann sich noch alles zum Guten wenden, wenn nicht etwa mißverständlich die Nahrungsmenge jetzt noch weiter gesteigert wird, um die ausbleibende $\mathrm{Zu}$ nahme auf diese Weise zu erzwingen. Ich erwähne diesen Punkt hier, meine Herren, weil ich auch in Verhältnissen, die eine verkehrte Ernährung und den Mangel an ärztlicher Ueberwachung erwarten lassen, eine relative Indikation zur natürlichen Ernährung sehe.

Aber es gibt noch andere Gefahren, denen auch die Säuglinge der besser gestellten Kreise bei künstlicher Ernährung ausgesetzt sind. Es handelt sich um die Rachitis und die sich mit ihr oft vereinigende Neigung zu Krämpfen. Wir können den Franzosen nicht darin beipflichten, daß die Ernährung mit guter Kuhmilch vor Rachitis schütze. Zuzugeben ist höchstens, daß eille zweckmäßig durchgeführte und ohne erhebliche Darmstörungen verlaufende künstliche Ernährung als solche nicht Rachitis erzeugt. Aber es wäre überhaupt eine falsche Fragestellung, ob künstliche Ernährung im Gegensatz zur natürlichen Rachitis erzeuge oder nicht. Die Neigung zur Rachitis ist vor allen Dingen erblich bedingt, und nur die Schwere der Rachitis ist es, die von Ernährungsstörungen vor allem subakuter und chronischer Art, von chronischen Infektionen (besonders tuberculöser Natur) und von ungünstigen hygienischen Verhältnissen (besonders vom Mangel an frischer Luft) bedingt ist. Daher findet sich auch bei Brustkindern nicht selten Rachitis, ohne aber einen gefährlichen Grad zu erreichen, und umgekehrt kann auch, wenngleich seltener, Rachitis bei Päppelkindern ganz oder bis auf Spuren vermißt werden. Insofern braucht die Gefahr einer Rachitis und der sich mit ihr kombinierenden Neigung zu Krämpfen nicht unter allen Umständen gegen die Einleitung der künstlichen Ernährung zu sprechen. Ist in der Familie schon ein Säugling bei der Flasche tadellos gediehen, so können sie die Flasche allenfalls auch bei dem folgenden Kinde wagen; ist Ihnen hingegen, meine Herren, das Vorkommen stärkerer Rachitis bei Verwandten, im besondern bei den Eltern und Geschwistern des Neugeborenen anamnestisch oder objektiv bekannt, so sollten Sie unter allen Umständen auf die natürliche Ernährung drin- gen. Die Gestaltung des Körperbaues und der Zähne gibt ebenso wie die motorische Entwicklung in der Familie oft untrügliche Hinweise auf die familiäre Verbreitung der Rachitis. Aber oft zeigen auch die hereditär belasteten Kinder selbst sofort nach der Geburt Zeichen von Rachitis. Im Gegensatz zu anderen Kinderärzten halte ich an dem Vorkommen einer k ongenitalen Rachitis fest, die sich in einer abnormen Nachgiebigkeit an den Rändern der Schädelknochen zeigt; hiermit kann sich ein Klaffen der Nähte und eine abnorme Größe der Fontanellen vereinigen; ohne eine abnorme Weichheit der Ränder würde aber dieser Befund für sich nicht für Rachitis verwertbar sein.

Muß schon die hereditäre Belastung mit Rachitis oder der Befund einer kongenitalen Rachitis an und für sich eine Anzeige für die Brusternährung geben, so ist dies besonders dann der Fall, wenn das Kind in der zweiten Hälfte des Kalenderjahres geboren wird. Ohne eine längere Periode des Genusses frischer Luft beginnt für die etwa im August oder später Geborenen im Herbst die lange Periode, in welcher die Witterung oder richtiger das Wohnungsklima ihre ungünstige Wirkung ausüben, sodaß ihnen bei gleichzeitiger künstlicher Elnährung die Rachitis besonders stark droht; die Schädlichkeit wirkt immer noch zwei bis drei Monate nach, sodal die Rachitis vom Januar bis in den Juni hinein am meisten in die Erscheinung tritt.

Man kann nicht sagen, daß die Uebererregbarkeit des Nervensystems, die im Stimmritzenkrampf, in allgemeinen Krämpfen und in den Erscheinungen einer larvierten oder manifesten Tetanie zutage tritt, unmittelbar von der Rachitis abhängig ist, aber jedenfalls kombiniert sie sich oft mit ihr; sie ist überhaupt viel häufiger als man noch bis vor kurzem wuBte. Was ich von der Rachitis sagte, gilt nun ebenso für die tetanoide Uebererregbarkeit, nur mit dem Unterschied, dab die Rachitis nicht in gleichem Maße eine unmittelbare Lebensgefahr bildet wie die Krämpfe; während jene in ihren leichteren Formen bei den Wohlhabenden oft übersehen wird und in der Tat von geringer praktischer $\mathrm{Be}$ deutung ist, läßt sich die schlimme Bedeutung der Krampfneigung bei arm und reich gleich wenig verkennen. Daher darf ich hier wohl noch sicherer auf Ihre Zustimmung rechnen, wenn ich für die von Krämpfen bedrohten Säuglinge dringend zur natürlichen Ernährung rate; durch sie sind die Säuglinge mit einer fast vollkommenen Sicherheit vor Stimmritzenkrampf und den verwandten Krämpfen geschützt. Wenn die Kinder im Alter von meist fünf und mehr Monaten ihre Krämpfe bekommen, ist es aber für eine Amme zu spät, sodaß Sie in der Regel auf dieses sicherste Mittel gegen immer neue Krämpfe verzichten müssen. Wenn in der Familie und im besondern bei älteren Geschwistern Stimmritzen- und andere Krämpfe vorgekommen sind oder wenn auch nur das familiäre Auftreten stärkerer Rachitis und damit die Wahrscheinlichkeit von Krämpfen besteht, so habe ich in meiner Praxis immer auf natürliche Ernährung gedrungen und hierdurch dem in der Familie dräuenden Unheil, welches oft ein Kind nach dem anderen hinrafft, erfolgreich begegnen können.

Schließlich wäre auch in diesem Zusammenhang noch ein besonderer Fall zu erwähnen, wo überhaupt immer die natürliche Ernährung angezeigt ist: die Frühgeburten und überhaupt die bei der Geburt nicht voll entwickelten Kinder. Auch wenn keine kongenitale Rachitis besteht, die übrigens oft hier sehr ausgesprochen ist, werden diese Kinder oft selbst bei natürlicher Ernährung, bestimmt aber in hohem Maße bei künstlicher Ernährung rachitisch und sterben dann häufig später an Krämpfen oder an gelegentlichen Infektionen (z. B. mit Influenza).

Nur kurz will ich, meine Herren, hier noch die Gefahr der Barlow schen Krankheit streifen, die an die künstliche Ernährung geknüpft ist. Sie läßt sich leicht vermeiden, wenn man die Milch nicht, wie es gerade früher die Wohlhabenden bei dem Soxhletschen Verfahren taten, zu lange erhitzt. Sie erinnern sich vielleicht, daß ich Sie vor wenigen Jahren auf das Bestehen einer Epidemie in Berlin aufmerksam machte, und diese darauf zurückführte, daß eine bestimmte Milch erst in der Molkerei und außerdem später im Hause der Erhitzung 
ausgesetzt werde. Daf ich mit meiner Erklärung im Gegensatz $\mathrm{zu}$ der Meinung anderer im Recht war, geht daraus hervor, daß die Epidemie auf meine Warnung hin schnell erlosch.

M. H.! In allen Spezialfällen, die ich soeben erwähnte, kann das Kind gut zunehmen, sodab wir ein vorzüglich ernährtes Kind vor uns haben. Und doch: welch ein Unterschied gegenüber gesunden Brustkindern! Oft weniger fett, sind die Brustkinder heiterer, frischer und beweglicher, muskelkräftiger und gegen alle Infektionen widerstandsfähiger. Ohne Zweifel hatten wir Aerzte in den letzten Jahrzehnten den Blick hierfür etwas eingebüßt, während uns neuerdings der Fortschritt in der Kenntnis der Säuglingskrankheiten und die erhöhte Möglichkeit, gesunde Brustkinder fortlaufend $\mathrm{zu}$ beobachten, die Erfolge der Brustkinder wieder besser erkennen läßt und im besonderen uns Kinderärzte veranlaßt, laut und nachdrücklich für alle Bevölkerungsklassen die natürliche Ernährung bis zur äußersten Möglichkeit zu predigen. Diese Aufgabe, die für die Verbesserung del Volksgesundheit sehr bedeutungsvoll ist, ist nicht unlösbar. Für die unbemittelte Bevölkerung wissen wir, daß der Rückgang des Stillens, von besonderen örtlichen Verhältnissen abgesehen, weder sozial noch physisch begründet ist. Das Stillen kommt hier einfach aus der Mode, weil man von seiner Wichtigkeit nicht mehr durchdrungen ist. Bei den verheirateten Frauen der unbemittelten Berliner Bevölkerung ${ }^{1)}$ sind nur 2,8\% durch Arbeit, 16,8\% durch Erkrankung, höchstens $4 \%$ durch Erkrankung der Neugeborenen am Stillen verhindert, und selbst wenn wir bei einem weiteren Viertel $(26,8 \%)$ ohne weiteres gelten lassen wollten, daß die Mütter keine Nahrung hatten, so bleiben noch immer $50 \%$, die ohne Grund vom Stillen absahen oder den Versuch aus unzureichenden Gründen bald wieder aufgaben. Wie viele Frauen in Berlin wirklich unter den gegebenen häuslichen Verhältnissen imstande wären, ihre Kinder - nötigenfalls unter Beifütterung - selbst zu stillen, läßt sich nicht angeben. Denn die Erfahrungen der Gebäranstalten, Wöchnerinnen- und Säuglingsheime sind nicht ohne weiteres auf die häuslichen Verhältnisse, die in so vielen Punkten schwieriger sind, zu übertragen. Aber jedenfalls wurden noch im Jahre 1885 in den Wohnungen von $1-2$ Zimmern $65 \%$ der Kinder vom ersten bis elften Lebensmonat an der Brust gefunden (in einem kleinen Teil mit Beinahrung ${ }^{2}$ ); da aber, wie wir wissen, auch in den achtziger Jahren ein Teil der Frauen aus nichtigen Gründen das Stillen unterließ, so können wir unbedenklich annehmen, daB $70-80 \%$ der Frauen in Berlin mehr oder weniger lange stillen könnten ${ }^{3}$ ). Demgegenüber finden wir in den Wohnungen von 4 und mehr Zimmern im Jahre 1900 weniger als $30 \%$ selbststillende Mütter.

Sind nun die bessergestellten Mütter so zart und kränklich, daß sie nicht öfter stillen können? Die Tatsache, daß der Zunahme des Wohlstandes die Abnahme des Stillens genau entspricht, läßt dies nicht eben wahrscheinlich erscheinen, und ebensowenig der dauernde Rückgang im Stillen, den ich schon früher erwähnte. Nehme ich die Erfahrungen der Praxis hinzu, so möchte ich folgendes behaupten. Das natürliche Bedürfnis zum Säugen und der Wunsch es auszuüben, ist in der Regel nur bei solchen Müttern lebhaft ausgesprochen, bei denen die Milchabsonderung früh und reichlich einsetzt. Bei anderen Frauen hingegen ist tagelang ein kräftiger Saugreiz nötig, der unter Umständen der Mutter Schmerzen macht und die Warze angreift, oder es besteht auch bei normaler Entwicklung der Sekretion eine starke allgemeine und örtliche Ueberempfindlichkeit (Empfindlichkeit der Warze, Stiche, Head sche Zonen am Rücken). Nach einigen Wochen hat sich die Mutter an das Stillen gewöhnt, und sie äußert höchstens noch den

1) Japha und Neumann, Die Säuglingsfürsorgestelle I der Stadt Berlin. Verlag S. Karger, 1906.

2) Gràtzer (aus Dr. H. Neumanns Kinderpoliklinik), Einiges über die Ernahrungsweise der Säuglinge bei der Berliner Arbeiterbevölkerung. Jahrbuch für Kinderheilkunde. N. F. Bd. 35 .

s) Diese Berechnung ist nur insofern nicht ganz einwandfrei, als bei der Volkszählung ein Teil der Flaschenkinder fehlte, soweit sie infolge ihrer hoheren Mortalität weggestorben waren. Die richtige Fragestellung wäre nicht: wie viele Säuglinge werden in einem gegebenen Augenblick gestillt?, sondern: wie viele von den Geborenen eines Jahres wurden gestillt? Diese Frage ist nicht genau zu beantworten.
Wunsch nach Zufütterung oder die Hoffnung auf eine nicht $\mathrm{zu}$ lange Fortführung des Stillens. Von seiten der Mutter wird meines Erachtens viel mehr aus körperlichen Beschwerden und falscher Beurteilung der Funktionsentwicklung der. Brustdrüse Widerstand geleistet, als infolge Abziehung durch den Beruf oder durch gesellschaftliche Verpflichtungen, oder infolge Oberflächlichkeit oder bösen Willen. Hieraus ergibt sich, welchen Einfluß die bei Geburt und Wochenbett beruflich tätigen Personen ausüben können, und da hier der Arzt in der Regel zugezogen wird, so hätte ich vor Ihnen, meine Herren Kollegen, offen die Frage zur Erörterung zu stellen: Is t der Arzt geneigt, dem Kinde sein natürliches Anrecht auf die Brust möglichst unverkürzt zuzugestehen, oder pflegt er sich bei seinem Rat zu stark durch die Rücksicht auf die Mutter leiten zu lassen - oft in einem solchen Maße, daß er nicht nur dem objektiven Befunde bei der Mutter, sondern auch den entferntesten Anzeigen gegen das Stillen schon im voraus Rechnung trägt? Diese Frage muß ich nach meiner Erfahrung leider bejahen und zwar in noch höherem Maße für den Geburtshelfer als für den praktischen Arzt: Sie sind nicht genügend von der Notwendigkeit der natürlichen Ernährung durchdrungen und darum wirkt ihr Rat auch, wo er das Stillen empfiehlt, oft nicht überzeugend. $W_{0}$ also im ungünstigen Fall wenigstens die ersten Wochen die Brust gegeben werden könnte oder dauernd gleichzeitig Brust und Flasche - und wer will dies im voraus sicher wissen? - wird in der Regel nach kurzem verzichtet - ganz zu schweigen von den Fällen, die bei gutem Willen aller Beteiligten ohne weiteres ganz glatt verlaufen würden.

Gestatten Sie, daß ich auf die Gegenanzeigen gegen das Stillen mit einigen Worten eingehe.

Bei schweren chronischen Krankheiten wäre auf das Stillen zu verzichten. Schlossmann läßt zwar selbst die Schwindsucht nicht als strenge Gegenanzeige beim Stillen gelten, indem er auf die Kräftigung und den Fettansatz bei manchen Stillenden hinweist. Ich selbst trage bei wenig ausgesprochener Lungentuberculose und bei besonderer Dringlichkeit der natürlichen Ernährung kein Bedenken, wenigstens die ersten Wochen stillen zu lassen.

Aber sehr viel öfter wird das Sängen untersagt, weil die Wöchnerin von den Geburtsblutungen zu erschöpft sei, weil sie überhaupt schwächlich, blutarm, bleichsüchtig, zart, nervös ist, weil sie beim Anlegen des Kindes Kreuz- und Rückenschmerzen oder Stiche hat. Die Erschöpfung, meine Herren, braucht in der Regel bei Frauen, die sich pflegen und schonen können, keinen Gegengrund zu geben, während eine puerperale Infektion mindestens einige vorbeugende Sorgfalt beim Anlegen erfordert. Die anderen Beschwerden, die die Mütter leicht auf die Lunge beziehen, können, soweit die objektive Untersuchung keine deutliche Begründung gibt, das Stillen kaum verbieten; sie werden bei einiger Körperpflege und einigem guten Willen allmählich überwunden. Da es an der Pflege nicht fehlt, so bleibt nur der gute und kräftige Wille zu erwecken, und hierzu steht uns Aerzten die psychische Beeinflussung zu Gebote, deren Bedentung für die Therapie meines Erachtens überhaupt nicht hoch genug eingeschätzt werden kann. Meine Herren, beruhigen Sie die Mutter über ihre Beschwerden, wenn Sie es mit gutem Gewissen tun können; versichern Sie ihr, daß das Stillen zuerst zwar einige Mühe und Beschwerden mache, weiterhin aber eine erfreuliche Gewohnheit werde, die der Mutter Genuß und Befriedigung gewährt. Erwarten Sie von der Mutter das Stillen als etwas Selbstverständliches, nicht als ein ungewöhnliches Opfer. Berichten Sie ihr, wie jedes Säugetier am besten gedeiht, wenn es von der eigenen Mutter gestillt wird, daf die Wissenschaft dies immer unwiderleglicher beweise, und daß neuerdings Reich und Arm entsprechend handelt; lassen Sie schließlich die Möglichkeit, eine Amme nehmen zu müssen, durchblicken - und, meine Herren, die natürliche Ernährung wird populär werden, und diese Popularität wird ihrerseits in ungeahnter Weise Ihre Bemühungen unterstützen.

Oft verzichtet man darauf, das Kind anzulegen, weil die Mutter offenbar keine Nahrung habe und man sie nicht unnütz 
quälen will. Aber man kann zwar aus der Untersuchung der Brust und aus der Tatsache, daß in der Familie immer gestillt wurde, auf Milch rechnen, ohne umgekehrt einer Brust eine gewisse Milchproduktion ohne weiteres absprechen zu können - es sei denn, daß man von einer früheren Entbindung eine sichere Erfahrung hätte. Seit der verdienstvollen Tätigkeit Schlossmanns haben wir gelernt, Brüsten Nahrung zu entlocken, wo man es früher nicht für möglich gehalten hätte, und wir gestalten diesen Nahrungsquell immer ergiebiger, seitdem wir in die Technik des Stillens immer tiefer eingedrungen sind. Läßt sich auch diese Technik vor allem in Anstalten ausüben, so ist doch auch im Hause viel zu erreichen. Vor allem sollte man zunächst die Brust, auch wenn sie noch wenig absondert, ausschlieblich geben, während man bei uns sofort mit einer Milchmischung bei der Hand ist und dadurch die Energie des Saugens bei dem Neugeborenen beeinträchtigt. Keinenfalls sollte man mit dem Anlegen bis zum Einschielien der Nahrung in die Brust warten, während man. ein normal entwickeltes Kind anderseits nicht früher anlegen sollte, als es sein Hungergefühl kräftig äußert. Selbst wenn es mehrere Tage dauert, bis der Saugreiz die Milch in die Brust zieht, so darf man doch auf den Erfolg warten, wenn man nur das Kind unter sorgfältiger täglicher Beobachtung behält. Bekommt der Neugeborene nach zwei Tagen noch keinen Tropfen Nahrung, so gebe man abgekochtes Zuckerwasser oder Fencheltee nach jedem Saugversuch, und nur bei schwächlichen Frühgeburien wird man schon frühzeitig daneben verdünnte Kuhmilch oder noch besser Ammenmilch reichen, weil sonst mit Erschöpfung der Kräfte auch die Stäpke des Saugens und hiermit die Möglichkeit des Milchzuflusses nachlassen würde. Im letzteren Fall ist es unter Umständen angezeigt, zunächst eine Amme mit ihrem Kind in das Haus zu nehmen und das kräftige Ammenkind bei der Mutter und das schwächliche neugeborene bei der Amme (mit Vermeidung zu großer Nahrungsaufnahme) anzulegen; natürlich muß die Amme außerdem mehrmals täglich ihr eigenes Kind stillen, damit sie nicht die Nahrung verliert.

Um dem Stillen einen guten Fortgang zu sichern, ist vor allem Ueberfütterung zu meiden. Lassen Sie einem Kind von normaler Entwicklung nicht nach einem bestimmten Schema die Brust reichen, sondern nur dann, wenn es deutliches Hungergefühl hat; es soll also überhaupt nicht - außer etwa $\mathrm{zu}$ der letzten Tagesmahlzeit - aus dem Schlaf genommen werden, wobei sich das Nahrungsbedürfnis schnell auf 4-6, meist auf 5 Mahlzeiten einzustellen pflegt. Wenn Sie die Mahlzeiten mit der Wage kontrollieren, müssen Sie bedenken, daß die Größe der einzelnen Mahlzeit schwankt, weniger jedoch die Tagesmenge der Nahrung.

Bei Mäßigkeit in der Ernährung können Sie mit einiger Sicherheit den Dyspepsien vorbeugen, welche auch an der Brust sehr häufig sind und leider sehr oft zum Unterbrechen des Stillens einen ziemlich unberechtigten Anlab geben. Die Stühle sind häufiger, gehackt und mit vielem Schleim, grünlich; dieser nicht vorschriftsmäßige Zustand darf allerdings nur dann Beunruhigung hervorrufen, wenn das Kind gleichzeitig wund wird, durch Unruhe Leibschmerzen verrät und nicht zunimmt, sondern auf dem gleichen Gewicht bleibt oder sogar abnimmt. Es liegt hier nun recht nahe, durch Annehmen einer Amme oder durch Abstillen diese Abnormität zu beseitigen: die Kuhmilch pflegt mit einem Schlage seltene und festere, oft zu feste Entleerungen herbeizuführen. Für die Zukunft des Kindes viel nützlicher scheint es mir aber, das Kind an der Mutterbrust zu lassen und durch eine Verminderung der Zahl oder Größe der Mahlzeiten in einigen Tagen oder Wochen zum Ziele zu kommen. Falls hierbei die Nahrungs menge zu gering wird, so ergänzt man sie durch dünne Schleimlösungen von Soxhlets Nährzucker $(5-10 \%)$, durch verdünnte Buttermilchsuppe oder durch eine gewöhnliche Milchmischung. Am zweckmäßigsten, aber in der Familie nur selten durchführbar, wäre es, da das Fett in der Frauenmilch die Beschwerden macht, dem Kind neben kleinen Mengen Muttermilch noch entsahnte Muttermilch zu reichen. In vielen Kurven könnte ich Ihnen zeigen; wie nach vorübergehendem Stillstand die Kinder dann bei der Mutter weiter gediehen.
M. H.! Oft wird das Stillen nicht ordentlich eingeleitet oder vorzeitig eingestellt. weil nach Ansicht der Mutter die Brust nicht genug Milch liefere. Indem durch die psychische Beunruhigung der Mutter die Sekretion wirklich beeinträchtigt wird und durch Beifütterung als solche durch die hierbei entstehenden dyspeptischen Beschwerden die Lust zum Saugen beim Kinde abnimmt, kann es tatsächlich zum Eingehen der Brust kommen. Die irrtümliche Annahme, zu wenig Milch zu produzieren, finden wir oft, selbst bei milchreichen Müttern, sei es, daß sie subjektiv das GefühI haben, sei es, weil das Kind viel schreit, oder die Gewichtszunahmen den theoretischen Anschauungen der Mutter nicht genügen. M. H.! Sie können hier den günstigsten Einfluß üben, indem Sie mit der Wage die getrunkenen Tagesmengen feststellen lassen und nach Ausschluß eines Hungerzustandes immer wieder betonen, daß ein ruhiges Kind, welches z. B. $120-150 \mathrm{~g}$ wöchentlich zunimmt, besser dran ist, als ein überfüttertes und unruhiges Kind. Nur, wenn jede suggestive Therapie, zu der ich unter den besseren Verhältnissen die Laktagoga rechne, versagt und die Nahrungsmengen und Gewichtszunahmen einige Zeit wirklich im Rückstand bleiben, würde ich Zufütterung in Gestalt einer Flasche, bei Kindern von sechs bis sieben Monaten in Form eines Breies gestatten.

Auf die Furcht, welche die Mütter mit Recht vor Schrunden und vor Brustdrüsenentzündungen haben, will ich hier nicht genauer eingehen. Sorgfältige Pflege der Warzen muß verhindern, daß an diesem Punkte das Stillen scheitert. Ohne andere Methoden zurückzusetzen, gehe ich selbst in folgender Weise vor: Die Brust wird nur einmal täglich gewaschen, wie es die Reinlichkeit gebietet. Das Kind wird ohne weiteres angelegt; nach dem Stillen wird reichlich mit Spiritus und Watte gewaschen und, nur sofern hierbei die ersten Spuren einer Schrunde durch Schmerzen bemerkbar werden, wird mit Naphthalan 2:1 Vasel. flav. eingesalbt; in diesem Fall wird vor dem nächsten Stillen dann die Salbe abgewischt, mit Spiritus gewaschen und mit Watte getrocknet. Ich glaube, daß die häufige Berührung mit Wasser die Warze mazeriert und beim Säugling das Auftreten von Soor begünstigt.

M. H.! Ich kann hier nicht auch die Ammenfrage aufrollen. Die Anzeigen für Ernährung durch die Amme bei Unfähigkeit der Mutter ergeben sich aus meinen obigen Ausführungen. Sie können der Mutter mit Recht die Amme als einen Ersatz vor Augen stellen, der moralisch und physisch gleich abstoßend ist. Mit der Verbesserung der Diagnostik finden wir die Syphilis bei Kindern von Müttern, die als Ammen gehen wollen, immer häufiger. Mit gutem Gewissen können Sie Ammen nur aus Säuglings- und Wöchnerinnenheimen nehmen, in denen Mutter und Kind während einiger Monate sorgfältig beobachtet sind; solche Anstalten, die sich mit Ammenabgabe neben ihren sonstigen Zielen befassen, sind aber sehr spärlich. $\left.{ }^{1}\right)$ Anderseits werden aber bei der Ammenwahl, wie ich nicht verschweigen kann, oft nicht nur überflüssige Forderungen ärztlicherseits gestellt, sondern auch gute Ammen durch fehlerhafte Technik schnell unbrauchbar gemacht. Wenn man z. B. erfährt, daß bei einem Säugling hintereinander acht Ammen genommen werden mußten, kann man getrost behaupten, daß dies mehr gegen die ärztliche Beratung als gegen die Ammen spricht.

M. H.! Ich weiß, daß ich Ihnen nichts Neues bieten konnte; die ganże moderne Bewegung fürs Stillen ist, wie alles, schon einmal dagewesen; aber ihr Erfolg wird diesmal nachhaltiger sein und die ganze Bevölkerung bis nach oben hin durchdringen, weil es sich nicht um Sentiments wie zur Zeit Rousseaus handelt, sondern um eine genauere Ergründung der Umstände, welche das Stillen gebieterisch verlangen und welche der Mutter die Erfüllung dieser Pflicht ermöglichen. 